\title{
TRANSIENT DYNAMICS OF THE KIDNEY DISEASE EPIDEMIC AMONG HIV-INFECTED INDIVIDUALS
}

\author{
DYLAN HULL-NYE*, BHAWNA MALIK*, RAVIKIRAN KESHAVAMURTHY*, AND ELISSA J. SCHWARTZ
}

\begin{abstract}
The prevalence of end stage kidney disease (ESKD) is rising among HIV-infected populations in several regions worldwide. We used an ordinary differential equation model of the dynamics of the AIDS and HIV+ ESKD populations to investigate the effect of antiretroviral therapy (ART) on the transient dynamics of the epidemic. We considered ART that blocks the entry to each population, by preventing individuals from joining the AIDS population and by reducing the development from AIDS to HIV + ESKD, as well as the combined effects together. Numerical simulation of our model revealed that at certain levels of ART below 100\%, the prevalence of HIV+ ESKD drops, but then increases again due to the recovery in the AIDS population. We then examined the dip in HIV+ ESKD seen with ART analytically by calculating the minimum HIV + ESKD level and the length of time to achieve this minimum. We also evaluated the minimum HIV+ ESKD level and its dependence on ART parameters, both singly and in combination. We conclude that our model predicts that the drop in HIV + ESKD prevalence seen after increased ART will be followed by an increase, unless ART is sufficiently high enough to eradicate HIV/AIDS.
\end{abstract}

\section{INTRODUCTION}

Acquired Immunodeficiency Syndrome (AIDS) is caused by Human Immunodeficiency Virus (HIV1), a retrovirus that affects the immune system by reducing CD4+ T cells, subsequently increasing the risk of opportunistic infections. By 2018, 37.9 million people were living with HIV/AIDS worldwide and more than 77 million people had died of the disease [28]. The black population is disproportionately affected by HIV/AIDS. Africa has more than two-thirds of global HIV/AIDS cases and has seen seven in ten deaths [14]. Similarly, African Americans, while comprising only $12 \%$ of the U.S. population, accounted for $44 \%$ of new HIV diagnoses in 2016 [3].

Kidney damage or reduced renal function that persists for more than 3 months is called chronic kidney disease (CKD) [7]. CKD is a significant non-infectious complication observed in HIV-infected patients $[1,2]$. Race is an important risk factor for CKD, which is more prevalent in black patients with HIV [26]. The population of those living with HIV/AIDS is at risk of developing end stage renal disease (ESRD, or also known as end stage kidney disease, ESKD), which is a result of impairment of renal function and kidney failure. Studies have shown a high rate of African Americans among those

Received by the editors 30 June 2020; accepted 17 December 2020; published online 24 December 2020.

2000 Mathematics Subject Classification. Primary 92-08; Secondary 37N25.

Key words and phrases. continuous ODE epidemic model, transient dynamics.

Portions of this research were undertaken as part of the CIMPA Summer School in Mathematical Biology in Kathmandu, Nepal, June 2019 with support from the Centre International de Mathmatiques Pures et Appliques (CIMPA), the International Centre for Theoretical Physics (ICTP) and the Society for Mathematical Biology (SMB).

*These authors contributed equally to this work. 
progressing from HIV + to HIV + ESKD [5]. The development of ESKD among individuals with AIDS has been increasing in recent years, and about $90 \%$ of those affected are of African descent [15].

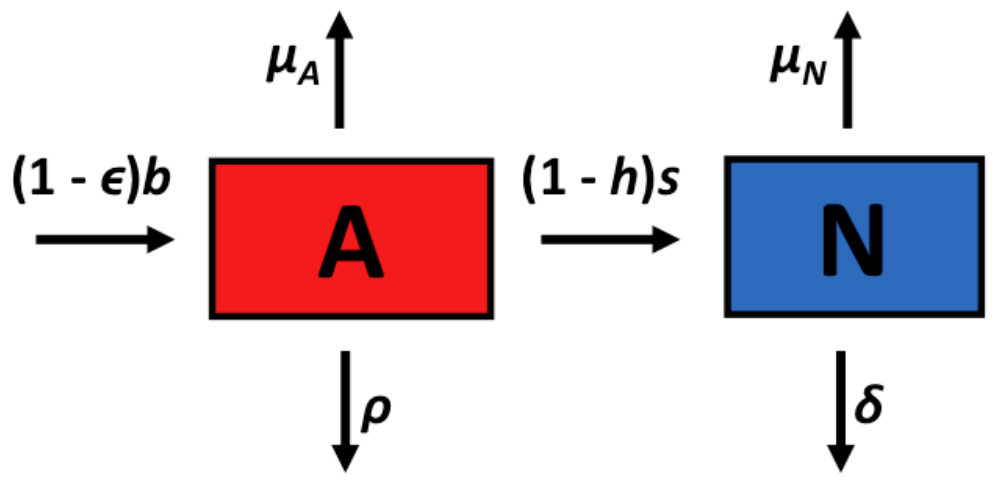

Figure 1. Flow diagram of AIDS and HIV+ ESKD dynamics. Individuals with AIDS $(A)$ progress to nephropathy $(N)$, or HIV+ ESKD. This occurs at rate $(1-h) s$, where $h$ represents the effectiveness of antiretroviral therapy (ART) in blocking progression to nephropathy. Recruitment to $A$ occurs at rate $(1-\epsilon) b$, where $\epsilon$ represents the effectiveness of ART in blocking the development of AIDS.

The availability of antiretroviral therapy (ART) and scaling up of these lifesaving drugs against HIV1 infection over the past 20 years is heralded as one of the biggest public health achievements of the century $[8,27]$. ART reduces the HIV viral load by inhibiting viral replication, transforming a highly fatal disease into a treatable, chronic infection [19]. According to the World Health Organization, in 2018, $62 \%$ of people living with HIV were receiving antiretroviral treatment [28]. Even though the rollout of ART has remained a challenge in low and middle-income countries, including in sub-Saharan Africa [27], it has substantially improved the prognosis of HIV-1 infection in recent years [24].

Relevant to kidney disease, ART has been associated with both short-term and long-term toxicities including nephrotoxicity $[12,13]$. However, when ART is employed along with the close monitoring of kidney function, it has been shown to effectively manage HIV-associated kidney disease. Importantly, studies have shown that effective treatment with ART decreases the morbidity and mortality in patients with HIV-associated kidney diseases and also the progression to ESKD [9, 17, 20], though the exact mechanisms by which ART ameliorates kidney disease are not yet clear [10], .

Here, we present a new epidemiological model of kidney disease development among HIV-infected individuals. Our goal is to analyze the transient dynamics of the model in order to predict the shortterm prevalence of disease. Specifically, we aim to understand the effects of ART on the HIV-infected population with kidney disease. Previous work [22] predicted that disease prevalence would rise due to the increase in the HIV-infected population, but the model utilized did not allow for the possibility to assess the effect of ART on this population. In this work, we add greater complexity to the population 
dynamics to permit this assessment. We also analyze the transient dynamics more closely, and we determine which conditions affect them.

\section{MODEL}

The model of kidney disease dynamics among HIV-infected individuals tracks two populations, AIDS $(A)$ and AIDS Nephropathy $(N)$ (also called HIV+ ESKD). The schematic diagram of the model is shown in Figure 1, and the model equations are as follows:

$$
\begin{aligned}
\frac{d A}{d t} & =(1-\epsilon) b-(1-h) s A-\rho A-\mu_{A} A \\
\frac{d N}{d t} & =(1-h) s A-\delta N-\mu_{N} N,
\end{aligned}
$$

with $A(0)=A_{0}$ and $N(0)=N_{0}$.

Briefly, new individuals with AIDS enter the $A$ population at a constant rate, $b$. These individuals die due to AIDS at rate $\rho$, or due to non-AIDS causes at rate $\mu_{A}$. Alternatively, they develop HIV+ ESKD at rate $s$. For now, we neglect individuals who develop HIV+ ESKD without developing AIDS. Individuals with HIV + ESKD die from the condition at rate $\delta$ or due to non-HIV + ESKD causes at rate $\mu_{N}$. Antiretroviral therapy (ART) is the standard of care for all individuals with HIV/AIDS, so we model its effects on populations $A$ and $N$ : ART that blocks individuals from developing AIDS is modeled by $\epsilon$; ART that blocks progression from AIDS to HIV + ESKD is modeled by $h$. Both $\epsilon$ and $h$ assume values between 0 and 1 to indicate ART efficacy between $0 \%$ and $100 \%$, respectively.

Our model improves upon the work of Schwartz et al. [22], in which populations grew without bound. This expansion was accomplished by separating the AIDS growth term into distinct entry and exit terms. We exclude the effects of ART on mortality in this study, in order to focus more specifically on the dynamics revealed by ART affecting $b$ and $s$. The list of model parameters is shown in Table 1 , along with the values for parameters and initial conditions used in numerical simulations. These values have been taken from the literature or fitted to CDC data, as described.

\section{Analytical Results}

3.1. Analytical Solution. Solving the model equations yields

$$
\begin{aligned}
& A(t)=\frac{(1-\epsilon) b}{k}+\left(A_{0}-\frac{(1-\epsilon) b}{k}\right) e^{-k t} \\
& N(t)=N_{0} e^{-k^{\prime} t}+\frac{(1-h) s}{k^{\prime}-k}\left(e^{-k t}-e^{-k^{\prime} t}\right)\left(A_{0}-\frac{(1-\epsilon) b}{k}\right)+\frac{(1-\epsilon) b(1-h) s}{k k^{\prime}}\left(1-e^{-k^{\prime} t}\right)
\end{aligned}
$$

where $k=(1-h) s+\rho+\mu_{A}$ and $k^{\prime}=\delta+\mu_{N}$.

3.2. Stability Analysis. There is one endemic equilibrium point $\left(A^{*}, N^{*}\right)$,

$$
\begin{aligned}
A^{*} & =\frac{(1-\epsilon) b}{\left[(1-h) s+\rho+\mu_{A}\right]} \\
N^{*} & =\frac{(1-h) s(1-\epsilon) b}{\left[\delta+\mu_{N}\right]\left[(1-h) s+\rho+\mu_{A}\right]} .
\end{aligned}
$$


TABle 1. Model Parameters

\begin{tabular}{|c|c|c|c|}
\hline Variables & Description & Initial Conditions & Source \\
\hline$A(t)$ & Population with AIDS & 31500 & {$[4,22]$} \\
\hline$N(t)$ & $\begin{array}{l}\text { Population with AIDS } \\
\text { Nephropathy } \quad(\mathrm{HIV}+ \\
\text { ESKD })\end{array}$ & 166 & {$[22]$} \\
\hline Parameters & Description & Values $\left(\right.$ year $^{-1}$ ) & Source \\
\hline$\epsilon$ & ART effectiveness on $b$ & Between 0 and 1 & Varies \\
\hline$s$ & $\begin{array}{l}\text { Rate of progression from } \\
\text { AIDS to HIV+ ESKD }\end{array}$ & 0.01 & {$[22]$} \\
\hline$h$ & ART effectiveness on $s$ & Between 0 and 1 & Varies \\
\hline$b$ & $\begin{array}{l}\text { Rate of entry to AIDS } \\
\text { population }\end{array}$ & 21970 & Fitted $^{a}$ \\
\hline$\rho$ & $\begin{array}{l}\text { Rate of death due to } \\
\text { AIDS }\end{array}$ & $1 / 6$ & $\begin{array}{l}\text { Based upon a } 6 \text { year lifespan } \\
\text { after AIDS diagnosis without } \\
\text { treatment }[6,11,23]^{b}\end{array}$ \\
\hline$\delta$ & $\begin{array}{l}\text { Rate of death due to } \\
\text { HIV + ESKD }\end{array}$ & 0.67 & $\begin{array}{l}\text { Based upon a } 1.5 \text { year lifespan } \\
\text { after diagnosis with AIDS and } \\
\text { HIV + ESKD with no treat- } \\
\text { ment }[25]\end{array}$ \\
\hline$\mu_{A}$ & $\begin{array}{l}\text { Natural mortality rate, } \\
\text { AIDS population }\end{array}$ & $1 / 55$ & $\begin{array}{l}\text { Based upon a } 55 \text { year lifespan } \\
\text { after infection }[16]\end{array}$ \\
\hline$\mu_{N}$ & $\begin{array}{l}\text { Natural mortality rate, } \\
\text { HIV+ ESKD population }\end{array}$ & $1 / 5$ & $\begin{array}{l}\text { Based upon a } 5 \text { year lifespan } \\
\text { after ESKD diagnosis }[18,21]^{c}\end{array}$ \\
\hline
\end{tabular}

${ }^{a}$ Fitted from the equation $A(t)$ and data [4] using other model values under the condition of no therapy.

${ }^{b}$ After AIDS diagnosis, individuals with no therapy survive approximately 6 years [6,11], with survival generally lower among black individuals [23].

${ }^{c}$ Average life expectancy on dialysis is 5-10 years; the lower bound was taken due to the additional condition of HIV infection and higher mortality seen in black individuals [18, 21].

The model has no basic reproductive number because there is no disease-free equilibrium. Eigenvalues of the Jacobian matrix are

$$
\begin{aligned}
& \lambda_{1}=-\left((1-h) s+\rho+\mu_{A}\right) \\
& \lambda_{2}=-\left(\delta+\mu_{N}\right) .
\end{aligned}
$$

Since the eigenvalues are negative, the equilibrium $\left(A^{*}, N^{*}\right)$ is stable. Moreover, for any initial conditions, the analytical solution approaches the equilibrium

$$
\begin{aligned}
& \lim _{t \rightarrow \infty} A(t)=A^{*} \\
& \lim _{t \rightarrow \infty} N(t)=N^{*},
\end{aligned}
$$

which gives us that $\left(A^{*}, N^{*}\right)$ is a globally asymptotically stable equilibrium point. 
3.3. Calculation of $N_{\min }$ and $t_{\min }$. Some ART efficacy values give rise to declining numbers in HIV+ ESKD followed by recovery to a steady state. We calculate the minimum value reached, $N_{\text {min }}$, as well as the length of time in which this minimum is reached, $t_{\min }$. This is accomplished by taking the derivative of the analytical solution for $N(t)$ (Equation 3.2), setting it to 0, and solving, yielding

$$
\begin{aligned}
t_{\text {min }}= & \frac{\ln (M)}{k-k^{\prime}} \quad M=\frac{(1-h) s\left(A_{0} k-b(1-\epsilon)\right)}{A_{0} k^{\prime} s(1-h)+N_{0} k^{\prime}\left(k-k^{\prime}\right)-b s(1-\epsilon)(1-h)} \\
N_{\text {min }}= & M^{\frac{k^{\prime}}{k^{\prime}-k}}\left(\frac{s(1-h)}{k-k^{\prime}}\left(A_{0}-\frac{b(1-\epsilon)}{k}\right)-\frac{s b(1-\epsilon)(1-h)}{k k^{\prime}}+N_{0}\right)+ \\
& M^{\frac{k}{k^{\prime}-k}}\left(\frac{s(1-h)}{k^{\prime}-k}\left(A_{0}-\frac{b(1-\epsilon)}{k}\right)\right)+\frac{s b(1-\epsilon)(1-h)}{k k^{\prime}} .
\end{aligned}
$$

\section{Numerical Results}

We performed numerical simulations of the epidemic trajectories under conditions representing various efficacies of antiretroviral therapy (ART). Values for parameters and initial conditions are shown in Table 1. First we show the epidemic trajectories with various levels of ART that decrease the progression from AIDS to HIV+ ESKD.

We investigated how much HIV+ ESKD $(N)$ would decrease initially, and then at steady state, with ART that decreased the progression from AIDS to HIV + ESKD $(h)$ by $0 \%, 70 \%, 80 \%, 90 \%$, or $100 \%$ (Figure 2). In the absence of ART $(h=0)$, both populations, AIDS $(A)$ and $\operatorname{HIV}+\operatorname{ESKD}(N)$, show epidemic trajectories that steeply grow and then level off to a plateau. When ART completely blocks the progression from AIDS to HIV $+\mathrm{ESKD}, h=1$. In this case, the AIDS $(A)$ population is unaffected (not shown), while the HIV $+\operatorname{ESKD}(N)$ population decreases to 0. When the degree to which ART prevents progression is $70 \%(h=0.7)$, HIV + ESKD decreases below 150 individuals in under 1 year, but then rises again to a steady state of 400 individuals by year 30 . With $80 \%$ effective ART $(h=0.8)$, HIV + ESKD decreases to 120 individuals in 1.5 years, and then rises again to a steady state of 270 individuals by year 15 . Therefore, just $10 \%$ more effective therapy can reduce the steady state level by $2 / 3$. When preventing progression is $90 \%$ effective $(h=0.9)$, HIV + ESKD decreases to a minimum just under 75 individuals in 2.5 years, rising to a steady state of 135 individuals. Thus, when ART effectiveness increases from $70 \%$ to $90 \%$, the steady state level of HIV+ ESKD decreases to $1 / 3$ of its previous level.

We observed that the level of HIV + ESKD $(N)$ decreased when ART was initially given, but then $N$ recovered and grew to a new steady state. This resurgence was due to the increase in the AIDS (A) population, which fuels the HIV + ESKD population (see inset, which shows the AIDS population increasing as the HIV+ ESKD population decreases). In other words, ART can block the development of HIV+ ESKD from AIDS, but this effect is not sustained if the risk pool that drives the HIV + ESKD epidemic (i.e., AIDS population $(A)$ ), is sufficient.

Thus, we next examined the effect of driving down the steady state of HIV + ESKD by reducing the risk pool (AIDS) with ART that blocks the AIDS entry rate (i.e., $\epsilon$ ). We simulated a block to the AIDS entry rate $(\epsilon)$ by $0 \%, 40 \%, 60 \%, 70 \%$, or $100 \%$ (Figure 2). In the absence of ART $(\epsilon=0)$, both populations, AIDS $(A)$ on the top right and HIV+ $\operatorname{ESKD}(N)$ on the bottom left, show epidemic trajectories that grow and then level off to a plateau. When ART completely blocks the entry to AIDS, 

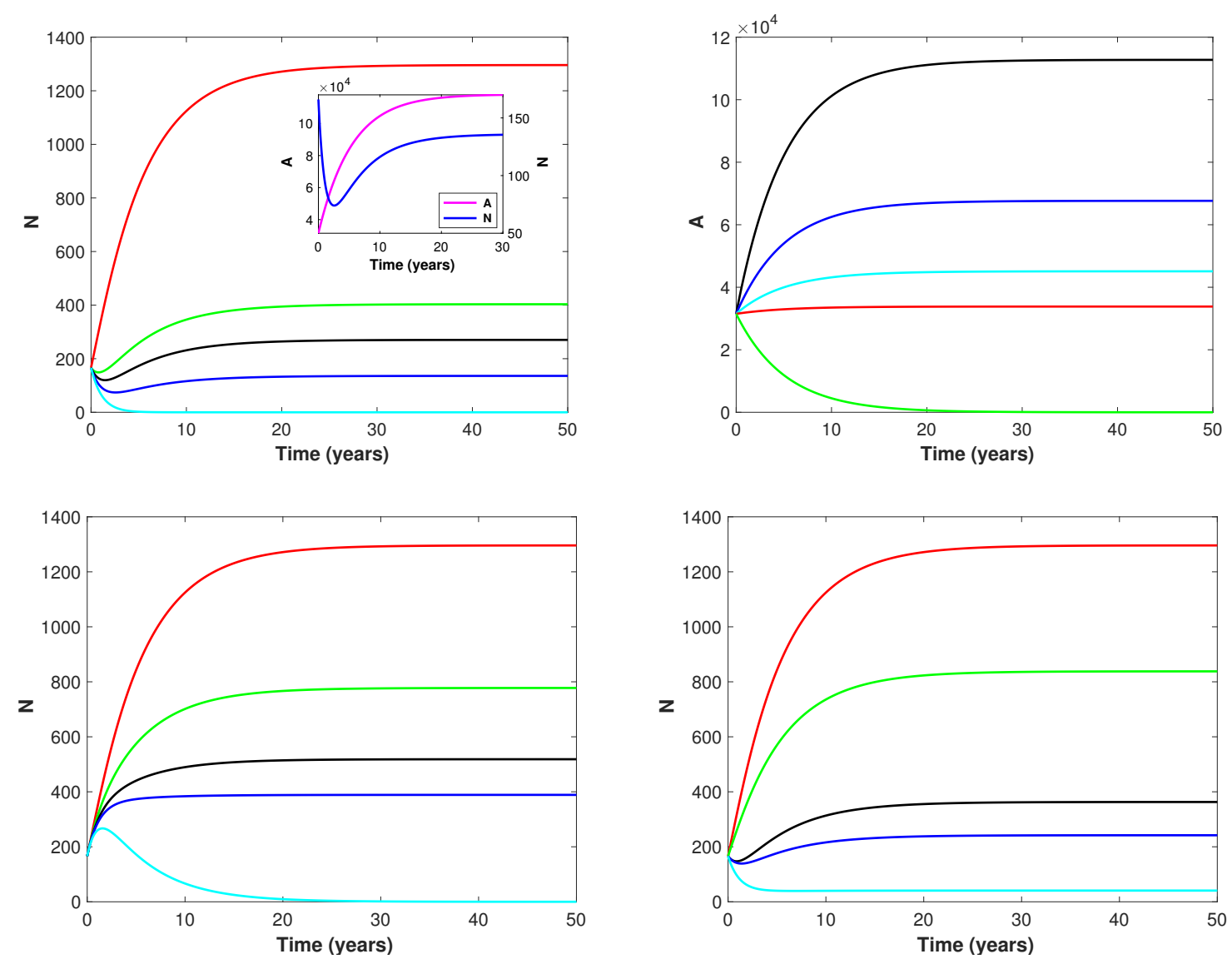

FIgURE 2. Model simulations showing the effects of antiretroviral therapy (ART). Top left: $N$ over time with $h=0$ (red), $h=0.7$ (green), $h=0.8$ (black), $h=0.9$ (blue), and $h=1$ (cyan). Other values are given in Table 1 with $\epsilon=0$. Inset: When $h=0.9$, $N$ is shown in blue and $A$ is shown in pink. Top right: $A$ over time with $\epsilon=0$ (black), $\epsilon=0.4$ (blue), $\epsilon=0.6$ (cyan), $\epsilon=0.7$ (red), $\epsilon=1$ (green). Other values are given in Table 1 with $h=0$. Bottom left: $N$ over time with $\epsilon=0$ (red), $\epsilon=0.4$ (green), $\epsilon=0.6$ (black), $\epsilon=0.7$ (blue), $\epsilon=1$ (cyan). Other values are given in Table 1 with $h=0$. Bottom right: $N$ over time with $h=\epsilon=0$ (red), $h=\epsilon=0.2$ (green), $h=0.7$ and $\epsilon=0.1$ (black), $h=0.7$ and $\epsilon=0.4$ (blue), $h=0.9$ and $\epsilon=0.7$ (cyan). Other values are given in Table 1.

$\epsilon=1$. In this case, both the AIDS $(A)$ population and the HIV $+\operatorname{ESKD}(N)$ population decrease to 0 . Interestingly, even with $100 \%$ effective ART $(\epsilon=1)$, the HIV $+\operatorname{ESKD}(N)$ population increases transiently before declining. With intermediate $\epsilon$ values, AIDS and HIV + ESKD increase less but still maintain positive steady states.

We then examined the potential effect on HIV + ESKD if ART blocks not only progression from AIDS to HIV + ESKD $(h>0)$, but simultaneously also blocks entry into the AIDS population $(\epsilon>0)$. The combined effect of ART acting at multiple points in the epidemic dynamics is seen here: When blocking progression is $90 \%(h=0.9)$ and blocking entry to AIDS is $70 \%(\epsilon=0.7)$, the steady state 
level of HIV+ ESKD is reduced to approximately 40 individuals (Figure 2, bottom right). Thus, in lieu of stronger therapy that effectively blocks a single mechanism, strong gains can be achieved when ART can simultaneously reduce entry into the AIDS population as well as reduce progression to HIV+ ESKD, even if its effectiveness at either is lower.
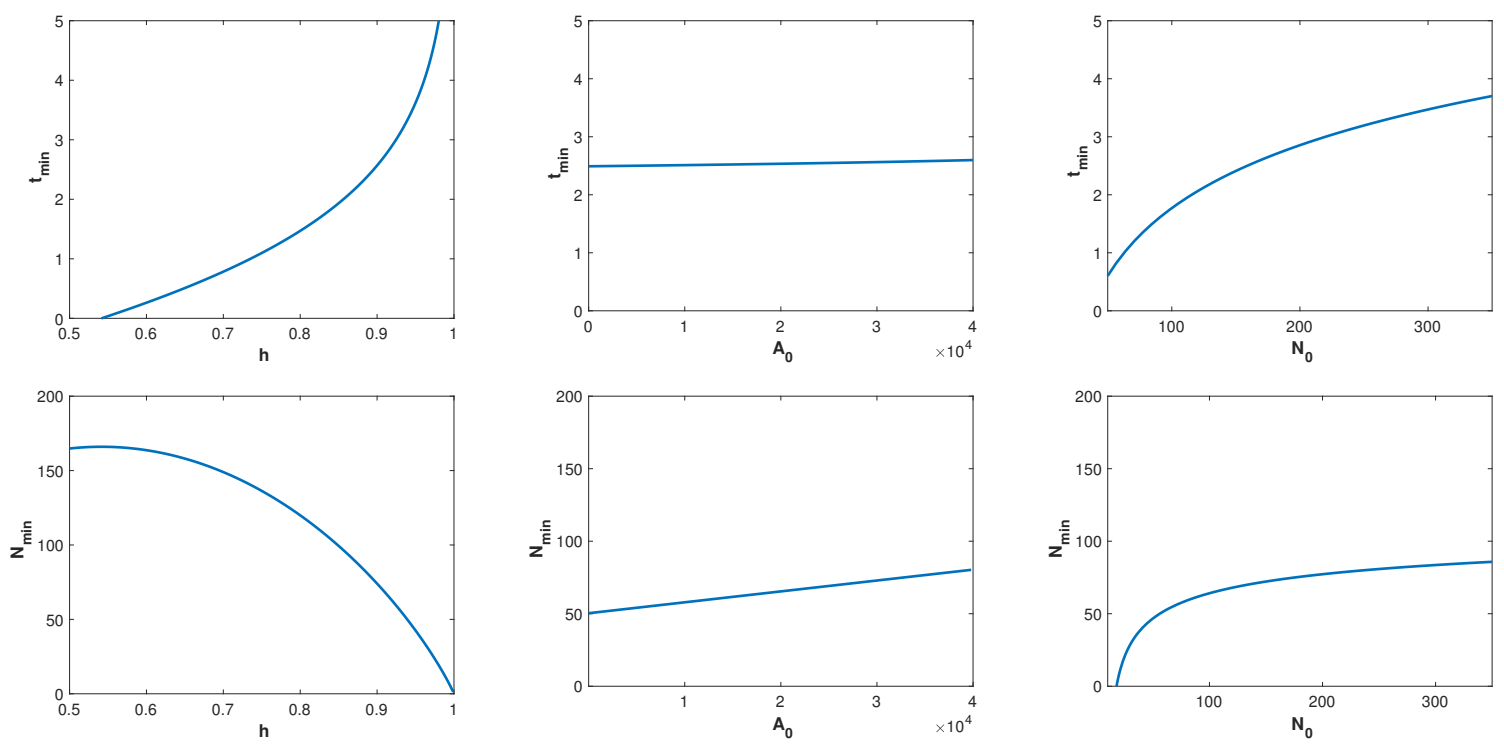

Figure 3. $t_{\min }$ (top) and $N_{\min }$ (bottom) as a function of $h$ (left), $A_{0}$ (middle), and $N_{0}$ (right). Left, $\epsilon=0$ and other values are as in Table 1 . Middle, $h=0.9, \epsilon=0$, and other values are as in Table 1 . Right, $h=0.9, \epsilon=0$, and other values are as in Table 1.

We note that different values of $h$ affect not only the steady state $N^{*}$ but also the values of $t_{\min }$ and $N_{\min }$. The closer $h$ is to 1 , the lower the minimum value of $N$, as would be expected, and the greater the minimum value of $t$. We show these minima across a range of values of $h$ from 0.5 to 1 (Figure 3, left). How the values of $t_{\text {min }}$ and $N_{\min }$ vary with initial conditions $A_{0}$ and $N_{0}$ are also shown (Figure 3 , middle and right, respectively). The initial AIDS population, $A_{0}$, has a negligible effect on $t_{\text {min }}$, and a larger value of $A_{0}$ gives a mildly higher $N_{\text {min }}$. The larger the initial size of the HIV + ESKD population, $N_{0}$, the later the $t_{\text {min }}$ is reached, and modestly, the higher the $N_{\text {min }}$. Therefore, low initial $N_{0}$ and $h$ around 0.6 give the earliest $t_{\min }$ values. For $N_{\min }$, lowest levels are seen with low $N_{0}$ values and high $h$.

Finally, we investigated the effects of ART and initial conditions on $N_{\min }$ more comprehensively. Figure 4 shows $N_{\min }$ given a range of values for $h$ and $A_{0}$ (top left), $h$ and $N_{0}$ (top right), and $h$ and $\epsilon$ (bottom left). The lowest $N_{\min }$ values occur when $h$ is high, particularly when $N_{0}$ is low. For all values across the surveyed ranges of $A_{0}$ and $\epsilon$, very high values of $h$ are needed to lower $N_{\text {min }}$. These figures show that to achieve a low $N_{\text {min }}, h$ has a stronger effect than $N_{0}, A_{0}$, and also $\epsilon$. In contrast, when we examined the effect of $h$ and $\epsilon$ on the steady state $N^{*}$, we found that $h$ and $\epsilon$ have equivalent efficacies on lowering $N^{*}$ (Figure 4, bottom right). Thus the differential effect of parameters $h$ and $\epsilon$ applies only to the transient dynamics. 

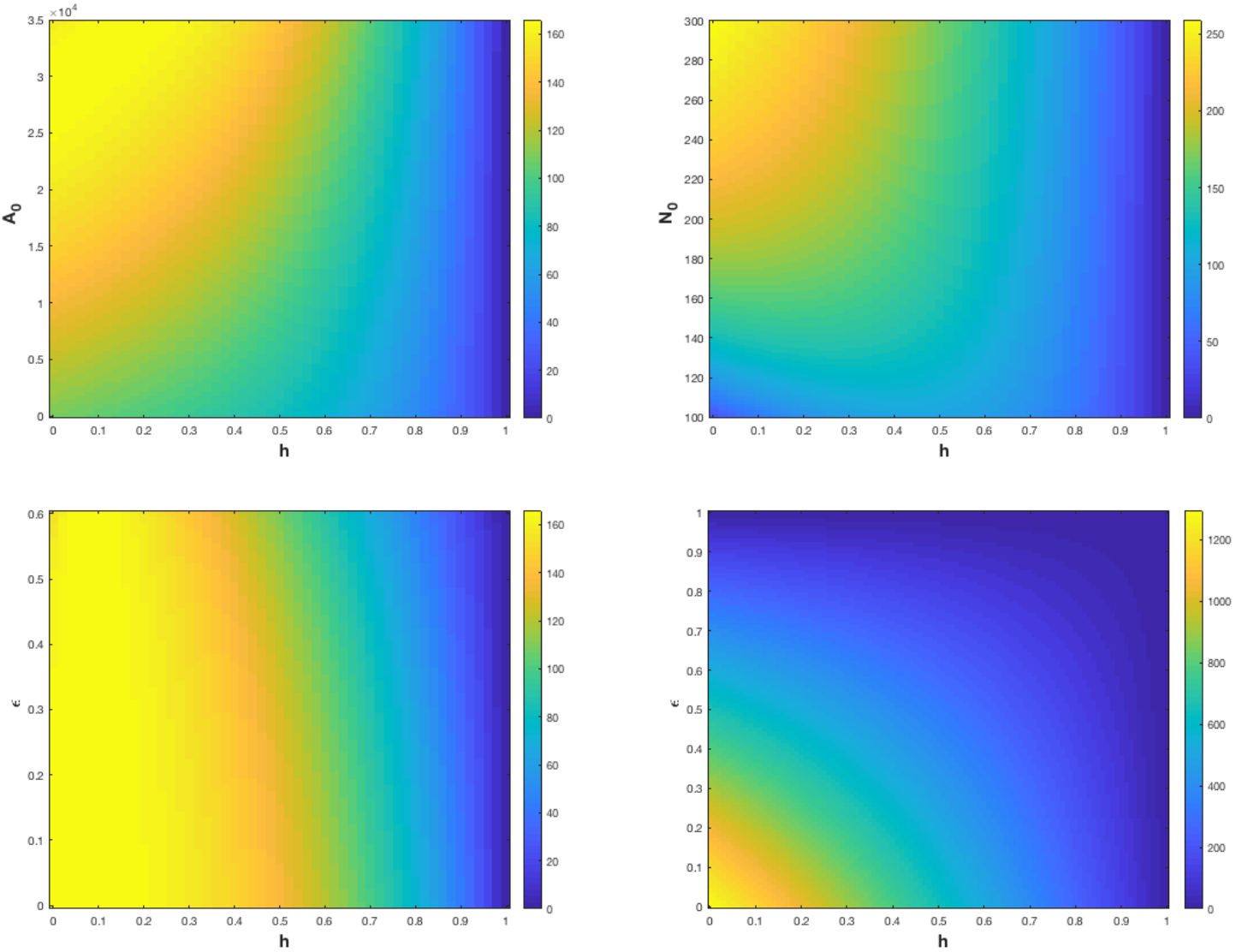

Figure 4. $N_{\min }$ values (from 0 in blue to 160 in yellow) shown according to how they vary with $h$ and $A_{0}$ (top left), $h$ and $N_{0}$ (top right), and $h$ and $\epsilon$ (bottom left). $N^{*}$ values (from 0 in blue to 1200 in yellow) shown according to how it varies with $h$ and $\epsilon$ (bottom right).

\section{Discussion}

This study demonstrates that when ART lowers HIV+ ESKD by blocking the progression from AIDS to HIV+ ESKD (via $h$ ), small improvements in $h$ efficacy can result in large reductions in prevalence. Blocking entry to AIDS with ART (via $\epsilon$ ) lowers HIV+ ESKD prevalence as well, even though its effect is indirect: it drives down HIV+ ESKD prevalence by reducing AIDS prevalence. The best outcomes occur with ART that targets entry to AIDS and progression to HIV+ ESKD simultaneously, even if the efficacy through each mechanism is lower.

Our simulations revealed that a dip in the prevalence of HIV + ESKD is seen with some ART values (e.g., $h=0.9$ ), followed by a rise to a lowered steady state. The minimum occurs earliest with values of $h$ around 0.6 and low $N_{0}$, and the smallest minima occur with high $h$ and low $N_{0}$. The value of $h$ has a stronger effect on the minimum $N$ than $N_{0}, A_{0}$ or $\epsilon$. However, practically speaking, it is important to note that the prevalence will rise again (unless ART is sufficiently high); this dip should not be viewed as an indication that the disease prevalence will continue to decrease, unless HIV/AIDS is eradicated. 
In future work, the model can be fitted with data sets from specific countries or regions affected by HIV + ESKD and used to estimate treatment levels needed to slow the increase of the epidemic in that region. Also, the model can be expanded to include additional complexity in the population dynamics, such as the early stages of HIV infection and the effects of ART on mortality rates. Finally, a complete uncertainty and sensitivity analysis would be useful for making predictions on future trends given variation in all parameters.

\section{Acknowledgements}

The authors would to thank Christina Cobbold, Rebecca Tyson, and Adriana Dawes for insightful suggestions and technical assistance on this manuscript. We would also like to thank Valerie Cheathon, Santosh Linkha, Dwarika Prasad Gautam, Nowraj Tiwari, and Ramesh Gautam for helplful contributions to a previous version of this work.

\section{REFERENCES}

[1] A. G. Abraham, K. N. Althoff, Y. Jing, M. M. Estrella, M. M. Kitahata, C. W. Wester, R. J. Bosch, H. Crane, J. Eron, M. J. Gill, M. A. Horberg, End-stage renal disease among HIV-infected adults in North America. Clin. Infect. Dis. 60(2015), 941-949.

[2] T. A. Adedeji, N. O. Adedeji, S. A. Adebisi, A. A. Idowu, M. B. Fawale, K. A. Jimoh, Prevalence and pattern of chronic kidney disease in antiretroviral-nave patients with HIV/AIDS. J. Int. Assoc. Provid. AIDS Care. 14(2015), 434440.

[3] Centers for Disease Control and Prevention. Health disparities in HIV/AIDS, viral hepatitis, STDs, and TB. https://www.cdc.gov/nchhstp/healthdisparities/africanamericans.html, Feb 2017, accessed April 19, 2019.

[4] Centers for Disease Control and Prevention, Division of HIV/AIDS Prevention, National Center for HIV, STD, and TB Prevention. HIV/AIDS surveillance report. Atlanta, 2002.

[5] S. Chaudhary, B. T. Workeneh, M. E. Montez-Rath, A.R. Zolopa, P. E. Klotman, W. C. Winkelmayer, Trends in the outcomes of end-stage renal disease secondary to human immunodeficiency virus-associated nephropathy. Nephrol. Dial. Transplant. 30(2015), 17341740.

[6] Collaborative Group on AIDS Incubation and HIV Survival including the CASCADE EU Concerted Action. Time from HIV-1 seroconversion to AIDS and death before widespread use of highly-active antiretroviral therapy: a collaborative re-analysis. Lancet. 355(2000), 1131-1137.

[7] J. Coresh, B. C. Astor, T. Greene, G. Eknoyan, A. S. Levey, Prevalence of chronic kidney disease and decreased kidney function in the adult US population: Third national health and nutrition examination survey. Am. J. Kidney Dis. 41(2003), 112.

[8] A. S. Fauci, R. W. Eisinger PEPFAR 15 years and counting the lives saved. N. Engl. J. Med. 378(2018), 314316.

[9] M. C. Foy, M. M. Estrella, G. M. Lucas, F. Tahir, D. M. Fine, R. D. Moore, M. G. Atta, Comparison of risk factors and outcomes in HIV immune complex kidney disease and HIV-associated nephropathy. Clin. J. Am. Soc. Nephrol. 8(2013), 1524-1532.

[10] X. Gao, A. Rosales, H. Karttunen, G. M. Bommana, B. Tandoh, Z. Yi, Z. Habib, V. DAgati, W. Zhang, M. J. Ross, The HIV protease inhibitor darunavir prevents kidney injury via HIV-independent mechanisms. Sci. Rep. 9(2019), 15857.

[11] K. M. Harrison, R. Song, X. Zhang, Life expectancy after HIV diagnosis based on national HIV surveillance data from 25 states, United States. J. Acquir. Immune Defic. Syndr. 53(2010), 124-130.

[12] J. Jao, C. M. Wyatt, Antiretroviral medications: Adverse effects on the kidney. Adv. Chronic Kidney Dis. 17(2010), $72-82$.

[13] R. Kalyesubula, M. A. Perazella, Nephrotoxicity of HAART. AIDS Res. Treat. 2011(2011), 562790.

[14] N. Kunene, M. Gella, T. Gala, Geographic controls of adult HIV/AIDS prevalence and their determinants for SubSaharan Africa countries. Am. J. Public Health, 5(2017), 130-137.

[15] S. K. Mallipattu, C.M. Wyatt, J.C.He, The new epidemiology of HIV-related kidney disease. J. AIDS Clin. Res. (2012), 001. 
[16] J. L. Marcus, C.R. Chao, W. A. Leyden， L.Xu， C.P. Quesenberry， D. B. Klein， W. J. Towner， M. A. Horberg, M. J. Silverberg, Narrowing the gap in life expectancy between HIV-infected and HIV-uninfected individuals with access to care, J. Acquir. Immune Defic. Syndr. 73(2016), 39-46.

[17] B. C. T. Mpondo, S. E. Kalluvya, R. N. Peck, R. Kabangila, B. R. Kidenya, L. Ephraim, D. W. Fitzgerald, J. A. Downs, Impact of antiretroviral therapy on renal function among HIV-infected Tanzanian adults: A retrospective cohort study. PLoS One. 9(2014), e89573.

[18] National Kidney Foundation, Dialysis Info. https://www.kidney.org/atoz/content/dialysisinfo, accessed Oct. 7, 2020.

[19] R. J. Pomerantz, D. L. Horn, Twenty years of therapy for HIV-1 infection. Nat. Med. 9(2003), 867-873.

[20] J. Prakash, M. Iqbal, P. S. Patel, S. Prakash, S. Sundar, U. Singh, Outcome of HIV related kidney diseases treated with combined antiretroviral therapy (cART). J. Assoc. Physicians India. 67(2019), 1822.

[21] R. Saran, B. Robinson, K. C. Abbott, L. Y. C. Agodoa, N. Bhave, J. Bragg-Gresham, R. Balkrishnan, X. Dietrich, A. Eckard, P. W. Eggers, A. Gaipov, US Renal Data System 2018 annual data report: Epidemiology of kidney disease in the United States, Am. J. Kidney Dis. 71(2018), 3.

[22] E. J. Schwartz, L. A. Szczech, M. J. Ross, M. E. Klotman, J. A. Winston and P. E. Klotman, Highly active antiretroviral therapy and the epidemic of HIV+ end stage renal disease, J. Am. Soc. Nephrol. 16(2005), 2412-2420.

[23] A. E. Siddiqi, X. Hu, H.I.Hall, Mortality among blacks or African Americans with HIV infection-United States, 2008-2012. Morbid. Mortal. Wkly. Rep. 64(2015), 81-86.

[24] UNAIDS, Joint United Nations Programme on HIV/AIDS. Global AIDS update 2016. Geneva; UNAIDS, 2016.(2019).

[25] US Renal Data System. USRDS 2002 Annual Data Report: Atlas of chronic kidney disease and end-stage renal disease in the United States. Bethesda, MD: National Institute of Health, National Institute of Diabetes and Digestive and Kidney diseases, (2002).

[26] US Renal Data System. USRDS 2007 Annual Data Report: Atlas of chronic kidney disease and end-stage renal disease in the United States. Bethesda, MD: National Institute of Health, National Institute of Diabetes and Digestive and Kidney diseases (2007).

[27] I. Weller, Delivery of antiretroviral therapy in Sub-Saharan Africa. Clin. Infect. Dis. 43(2006), 777778.

[28] World Health Organization, Report on the global HIV/AIDS epidemic. http://www.who.int/hiv/en/, accessed June 29, 2019.

Department of Mathematics \& Statistics, Washington State University, Pullman, Wa, USA

E-mail address: dylan.hull-nye@wsu.edu

Department of Mathematics, Shiv Nadar University, Dadri, India

E-mail address: bm650@snu.edu.in

Paul G. Allen School for Global Animal Health, Washington State University, Pullman, Wa, USA

E-mail address: r.keshavamurthy@wsu.edu

Corresponding author, Department of Mathematics \& Statistics and School of Biological Sciences, Washington State University, Pullman, WA, USA

E-mail address: ejs@wsu.edu 